\title{
Caracterização por ressonância magnética nuclear do sistema poroso de coquinas da Formação Morro do Chaves, Bacia Sergipe-Alagoas.
}

Fernanda O. Hoerlle, William G. A. L. Silva, Edmilson H. Rios, Maira C. O. Lima, Elizabeth M. B. D. Pontedeiro, José L. D. Alves, Paulo Couto

Programa de Engenharia Civil, COPPE/Universidade Federal do Rio de Janeiro

Copyright 2018, SBGf - Sociedade Brasileira de Geofísica

Este texto foi preparado para a apresentação no VIII Simpósio Brasileiro de Geofísica, Salinópolis, 18 a 20 de setembro de 2018. Seu conteúdo foi revisado pelo Comite Técnico do VIII SimBGf, mas não necessariamente representa a opinião da SBGf ou de seus associados. É proibida a reprodução total ou parcial deste material para propósitos comerciais sem prévia autorização da SBGf.

\section{Resumo}

A ressonância magnética nuclear (RMN) é uma ferramenta bastante utilizada na indústria de petróleo para a caracterização de rochas reservatório. Neste trabalho estudou-se o sistema poroso de coquinas da Formação Morro do Chaves, da Bacia Sergipe-Alagoas, considerada como possível análogo à reservatórios de coquinas no Pré-sal. Objetivou-se realizar a caracterização do sistema poroso dessas rochas em relação à quantificação da porosidade total e distribuição do tamanho de poros. Como conclusão verificou-se a heterogeneidade das coquinas e determinou-se o particionamento do sistema poroso dessas rochas em macro, meso e micro poros.

\section{Introdução}

A RMN é uma técnica muito importante que investiga as propriedades físicas dos materiais. Especialmente empregada na indústria de petróleo, é utilizada com frequência em laboratório e em campo para auxiliar na caracterização de reservatórios (Rios et al., 2010; Polli, 2016). Para a aquisição dos dados de $\mathrm{RMN}$, a rocha precisa estar saturada com fluido (água salina ou doce, hidrocarboneto liquido ou gasoso), uma vez que o sinal adquirido é proveniente da magnetização dos spins dos prótons contido no fluido.

Neste trabalho empregou-se a técnica de RMN para a obtenção do tempo de relaxação transversal $\left(T_{2}\right)$ de rochas carbonáticas classificadas como coquinas. A partir desses dados, objetivou-se avaliar a porosidade particionada segundo a classificação de Lønøy (2006), aplicando a relaxatividade superficial calculada por De Luna et al. (2016), parâmetro aplicado para converter os valores de $\mathrm{T}_{2}$ para raio de poro. Com isso, obteve-se a distribuição de tamanho de poros em plugues de amostras que apresentam o sistema poroso bastante heterogêneo.

Analisou-se nove amostras de coquinas pertencentes à Formação Morro do Chaves, Bacia Sergipe-Alagoas, nordeste do Brasil. Tratam-se de rochas formadas por bioclastos, principalmente por bivalves lacustres, que sofreram transporte e foram afetados por diversas fases de diagênese, acarretando em um sistema poroso complexo (Corbett et al., 2016).

Para a aquisição do sinal de RMN aplica-se, nas amostras saturadas, um campo magnético constante $\left(B_{0}\right)$ que causa o alinhamento dos spins dos prótons, isótopo ${ }^{1} \mathrm{H}$ presente nos fluidos de reservatório, em relação ao campo magnético externo. Posteriormente, aplica-se um campo magnético oscilante $\left(B_{1}\right)$, perpendicular a $B_{0}$, com o intuito de tirar os prótons do equilíbrio. Quando se remove o segundo campo magnético, os spins tendem a retomar, ou relaxar, para a direção original que haviam sido alinhados. Esse processo é conhecido como relaxação magnética nuclear e o sinal elétrico, medido por indução eletromagnética, chama-se free induction decay (FID), sendo modelado por decaimentos exponenciais. Experimentalmente, esse decaimento é adquirido pela técnica CPMG e o sinal ajustados por múltiplas exponenciais, cada qual com um valor de amplitude e taxa de decaimento $1 / T_{2}$, em que $T_{2}$ é uma constante de tempo de relaxação transversal, também chamado de interação spin-spin. As amplitudes do sinal em função do tempo de relaxação são obtidas por inversão numérica.

Segundo Coates et al. (1999), o sinal de $\mathrm{T}_{2}$ pode ser relacionado à distribuição de tamanho de poros, porosidade e tipo de fluido presente nos poros. $\mathrm{O} \mathrm{T}_{2}$ é diretamente proporcional à razão superfície-volume do poro, que é uma medida do tamanho do poro quando a formação está $100 \%$ saturada com um único fluido de salmoura e utiliza-se um curto espaço inter-eco. Poros grandes são associados à tempos de relaxação mais longos, enquanto poros pequenos estão associados à tempos de relaxação mais curtos.

\section{Metodologia}

Anteriormente à aquisição de dados, as nove amostras estudadas (1-2A, 1-4, 1-9A, 1-14A, 1-18B, 1-19B, 1-28, 1$31 \mathrm{~B}, 1-34 \mathrm{~A}$ ) foram limpas com tolueno e metanol, para a remoção de óleo residual e sais contidos nos poros. Para a realização da análise de RMN, saturou-se as amostras com salmoura de concentração 50.000ppm de $\mathrm{NaCl}$ e densidade de $1,04 \mathrm{~g} / \mathrm{cm}^{3}$ a $25^{\circ} \mathrm{C}$, medida no Density Meter DM40 (Mettler Toledo). A salmoura foi confeccionada no Laboratório de Recuperação Avançada de Petróleo (LRAP-COPPE/UFRJ) e a saturação, no Laboratório de Petrofísica do Cenpes.

Ao final do procedimento de saturação, pesou-se as amostras para verificar a eficácia do procedimento. Usualmente, espera-se $100 \%$ de saturação das amostras, porém se aceita um erro de $\pm 5 \%$ (RP40, 1998). Empregou-se as seguintes equações para se calcular 0 índice de saturação em porcentagem:

$$
\begin{gathered}
V_{\text {salmoura }}=M_{\text {saturada }}-M_{\text {seca }} \rho_{\text {salmoura }} \\
I S=V_{\text {salmoura }} / V_{p}
\end{gathered}
$$


em que IS é o índice de saturação, Vsalmoura é o volume de salmoura saturando o espaço poroso da rocha, Vp é o volume de poros da amostra, Msaturada é a massa da amostra saturada em salmoura, Mseca é a massa seca da amostra, Psalmoura é a densidade da salmoura,

Para a aquisição do sinal de RMN utilizou-se o equipamento de baixo campo da Oxford Instruments, MQC - $5 \mathrm{MHz}$, pertencente ao Laboratório de Ressonância Magnética Nuclear de Baixa Resolução, do Instituto de Macromoléculas (IMA-UFRJ). Obteve-se os decaimentos da relaxação magnética transversal através da sequência de pulsos CPMG (Coates et al., 1999) para o núcleo de ${ }^{1} \mathrm{H}$.

Realizou-se a transformação do sinal de $\mathrm{T}_{2}$ (em microssegundos) para a escala de distância (em micrômetros) através da equação:

$$
1 / T_{2, \text { superficie }}=\rho_{2} \times S / V
$$

em que a constante $\rho_{2}$ é a relaxatividade superficial e $S / V$ é a razão área superficial por volume.

A aquisição dos dados de porosidade de RMN foram comparados com dados de porosidade obtidos através da técnica de expansão de gás He em que a Lei de Boyle é aplicada. Utilizou-se o porosímetro DV-4000 da Weatherford, pertencente ao LRAP-COPPE/UFRJ. Lucia (2007) indicou esse método como o mais preciso para tal mensuração e, por esse motivo, utilizou-se dele como base de comparação para as medidas de porosidade obtida com a RMN.

\section{Resultados}

Os resultados de índice de saturação são apresentados na Tabela 1. Esse procedimento foi satisfatório para todas amostras, exceto para a amostra 1-34A, que teve saturação de apenas $75,08 \%$ do espaço poroso. De Luna et al. (2016) afirmou que poros do tipo vugular podem diminuir a eficácia do processo de saturação por não conseguirem reter a solução salina. Como o sinal de RMN é proporcional ao volume de fluidos é esperado um déficit na porosidade obtida para essa amostra.

Tendo sido feita a aquisição do sinal de RMN, calculouse a porosidade das amostras. $O$ resultado, juntamente com a comparação da porosidade de $\mathrm{He}$ e o erro relativo entre as duas medidas, encontra-se na Tabela 1 e na Figura 1. O coeficiente de determinação $\left(R^{2}\right)$ entre as porosidades de RMN e do porosímetro é de 0,9063, demonstrando que a RMN é um bom método para obtenção dessa propriedade.

Com a análise dos dados de RMN, além da obtenção da porosidade, também pôde-se determinar a distribuição do tamanho de poros. Para tanto, o sinal de RMN medido foi convertido na distribuição de $T_{2}$ através da Transformada Inversa de Laplace e um gráfico de porosidade incremental versus $\mathrm{T}_{2}$ foi gerado (Fig. 2). Esse gráfico pode ser interpretado como a distribuição de tamanho de poros das rochas (Westphal, 2005). Entretanto, para se ter uma medida mais precisa, transformou-se a escala de tempo de $T_{2}$ para a escala de distância, ou seja, para escala de tamanho de raio de poro. Assim, confeccionou- se gráficos de distribuição de tamanho de poros, em microssegundos versus porosidade incremental Figura 3 e Figura 4.

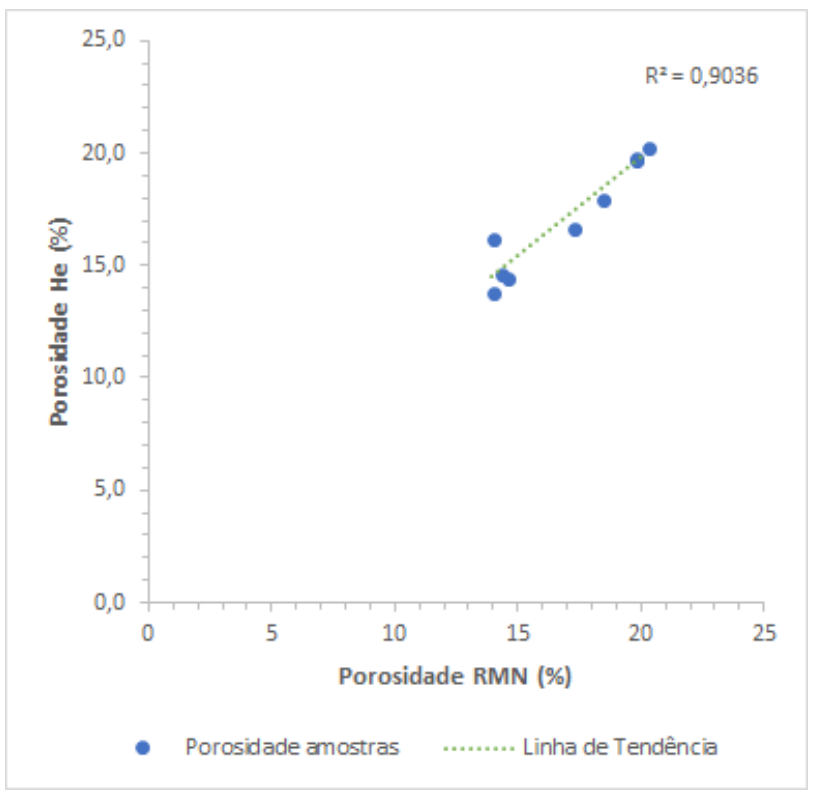

Figura 1 - Correlação entre porosidade a gás e porosidade por RMN.

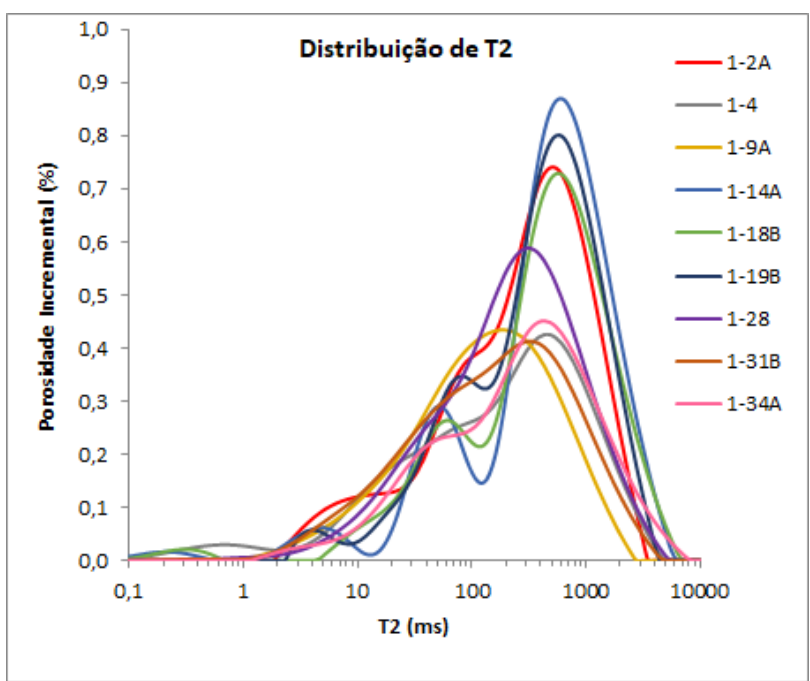

Figura 2 - Distribuição de $T_{2}$.

De Luna et al. (2016) encontraram um valor médio de relaxatividade superficial de $35,76 \mu \mathrm{m} / \mathrm{s}$ para cinco amostras da mesma Camada 2B da Formação Morro do Chaves. Portanto, utilizou-se esse valor médio para tal transformação dos dados de RMN.

Neste trabalho empregou-se a classificação modificada de Lønøy (2006) para particionamento da porosidade por RMN: poros abaixo de $50 \mu \mathrm{m}$ são considerados microporos, 50-100 $\mu \mathrm{m}$ mesoporos e acima de $100 \mu \mathrm{m}$ macroporos. A partir da porosidade acumulada da distribuição de T2 quantificou-se a porosidade particionada e sua porcentagem relativa (Tab. 2). 


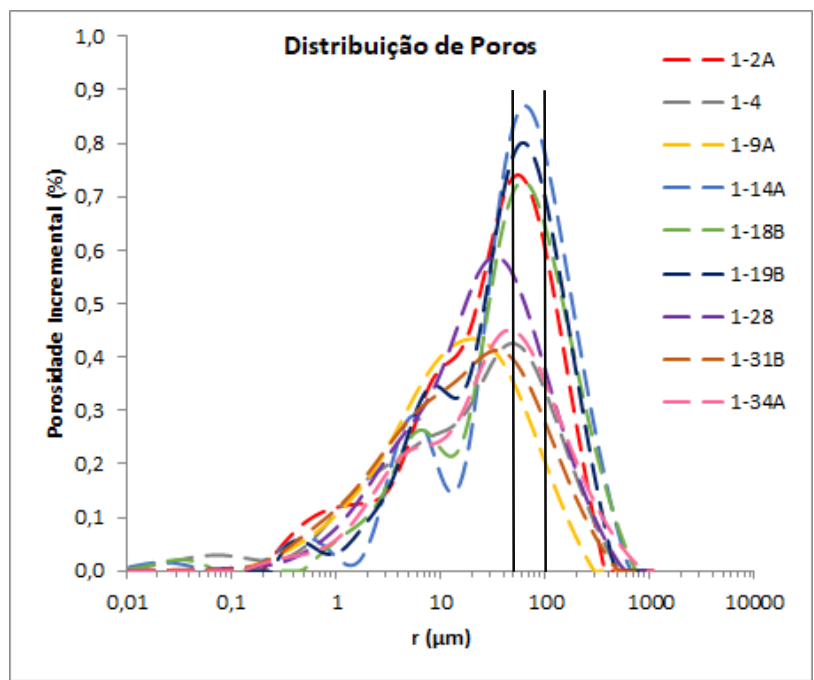

Figura 3 - Distribuição de tamanho de poros.

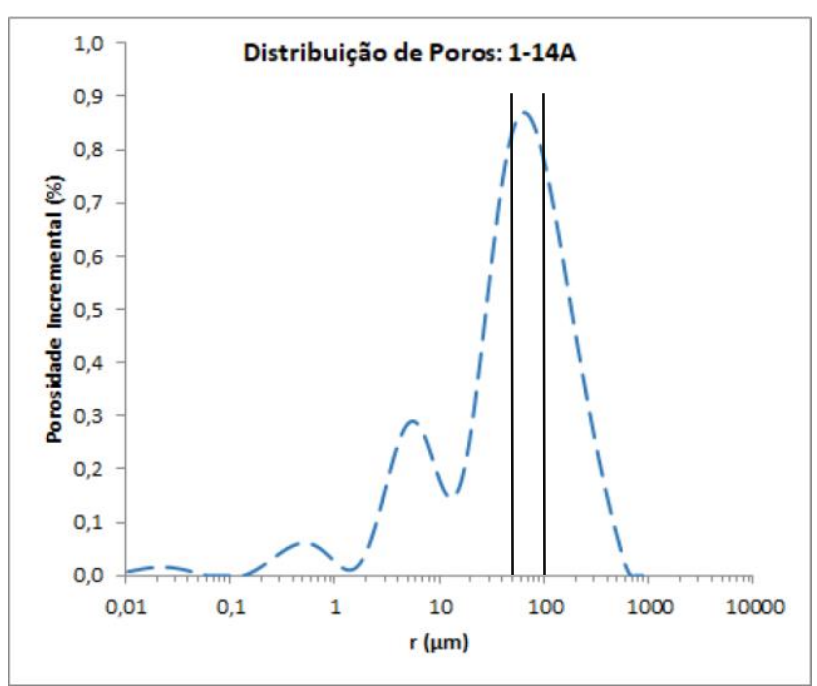

Figura 4 - Distribuição de tamanho de poros para a amostra 1-14A.

Observou-se que nas amostras 1-2A, 1-4, 1-9A, 1-28, 1$31 \mathrm{~B}$ e 1-34A a maioria dos poros é representada pelos microporos, seguido dos macroporos e, em menor representatividade, mesoporos. No entanto, os plugues $1-14 \mathrm{~A}, 1-18 \mathrm{~B}$ e $1-19 \mathrm{~B}$ apresentaram o particionamento diferente das demais amostras, sendo a maioria dos poros representados por macroporos, seguidos por microporos e, em menor porcentagem, mesoporos (Tab. 2). Para todas as amostras os mesoporos representam a menor quantidade dos poros, com exceção da 1-9A, em que os macroporos e mesoporos aparecem em porcentagens semelhantes.

\section{Discussão e Conclusões}

A técnica de RMN em laboratório mostrou-se um método eficiente na medida de porosidade para rochas do tipo coquina, pertencentes à Formação Morro do Chaves. O particionamento da porosidade segundo a classificação de Lønøy (2006) e a relaxatividade superficial calculada por Luna et al. (2016) também se mostraram satisfatórios.

A partir dos dados e gráficos de distribuição de tamanho de poros foram observados a heterogeneidade das coquinas. As amostras são compostas por poros do tipo micro, meso e macro.

Com as curvas normalizadas de porosidade acumulada, pôde-se concluir que essas rochas não apresentam um padrão predominante de tamanho de poros. Notou-se que $66,7 \%$ das amostras estudadas têm a maioria dos poros representada pelos microporos, seguido dos macroporos e, por último, mesoporos. Por sua vez, os $33,3 \%$ restantes possuem maioria dos poros representados pelos macroporos, seguidos dos microporos e, em menor porcentagem, os mesoporos.

\section{Agradecimentos}

Os autores gostariam de agradecer ao patrocínio da Shell Brasil, Petrobras e CNPq. Agradecemos à Gerência da Caracterização de Rochas e Fluidos/Pesquisa e Desenvolvimento em Exploração e Produção do Cenpes/Petrobrás, pelo auxílio na saturação das rochas. Agradecemos ao IMA-UFRJ, em especial a Profa. Maria Inês Bruno Tavares, por ter disponibilizado o equipamento de RMN para a aquisição dos dados. Nosso obrigado também às equipes de pesquisadores LRAP/COPPE/UFRJ e do Laboratório de Geologia Sedimentar (Lagesed-UFRJ) pelo auxílio durante a pesquisa. Agradecemos à cessão das amostras do Lagesed pelo projeto SACL - Análise geológica sedimentar de sucessões carbonáticas cretácicas em uma bacia sedimentar brasileira (ANP autorização n.551/2012; BG E\&amp;P Brasil projeto BG-04; Fundação COPPETEC projeto IGEO-15.981).

\section{Referências}

Coates, G. R., Xiao, L., \& Prammer, M. G., 1999, NMR logging: principles and applications. Houston: Haliburton Energy Services.

Corbett et al. (5 coautores), 2016. Integration of cretaceous Morro do Chaves rock properties (NE Brazil) with the Holocene Hamelin Coquina architecture (Shark Bay, Western Australia) to model effective permeability. Petroleum Geoscience, v. 22, n. 2, p. 105-122.

Hoerlle, F.O. Estudo da heterogeneidade, anisotropia e conectividade do sistema poroso das coquinas da Formação Morro do Chaves (Bacia de Sergipe-Alagoas). 2018. 142f. Dissertação de Mestrado (Mestrado em Engenharia Civil) - COPPE, Universidade Federal do Rio de Janeiro.

Lønøy, A., 2006. Making sense of carbonate pore systems. AAPG bulletin, v. 90, n. 9, p. 1381-1405.

Lucia, F. J., 2007. Carbonate reservoir characterization: An integrated approach. Springer Science \& Business Media. 
de Luna et al. (06 coautores), 2016. Petrophysical rock typing of coquinas from the morro do chaves formation, sergipe-alagoas basin (northeast brazil). Revista Brasileira de Geofísica, v. 34, n. 4.

Polli, R. S., 2016, Estudo do processo de acidificação de rochas reservatório por meio de RMN e microtomografia de raios-X. Tese de D.Sc., USP, São Carlos, SP, Brasil.

Rios et al. (05 coautores), 2010. Caracterização petrofísica de rochas reservatório por Ressonância Magnética Nuclear. In: IV Simpósio Brasileiro de Geofísica.

RP40, A. P. I., 1998. Recommended practices for core analysis.

Westphal, H. et al (4coautores), 2005. NMR measurements in carbonates rocks: problems and an approach to a solution. Pure and Applied Geophysics, n.162, p.549-570.

Tabela 1 - Dados de índice de saturação, porosidade de RMN e porosidade He.

\begin{tabular}{|c|c|c|c|c|c|c|c|}
\hline Amostra & $\begin{array}{l}\text { Massa } \\
\text { Seca } \\
(\mathrm{g})\end{array}$ & $\begin{array}{l}\text { Massa } \\
\text { Saturada } \\
\text { (g) }\end{array}$ & $\begin{array}{c}\text { Densidade } \\
\text { Salmoura } \\
\text { (g/cc) }\end{array}$ & $\begin{array}{l}\text { Volume } \\
\text { Poroso } \\
\text { (cc) }\end{array}$ & $\begin{array}{c}\text { Índice de } \\
\text { Saturação } \\
(\%)\end{array}$ & $\begin{array}{c}\text { фRMN } \\
(\%)\end{array}$ & $\begin{array}{c}\phi \mathrm{He} \\
(\%)\end{array}$ \\
\hline $1-2 A$ & 75,91 & 82,94 & 1,04 & 6,98 & 96,99 & 19,7 & 19,9 \\
\hline $1-4$ & 82,21 & 87,49 & 1,04 & 5,26 & 96,67 & 14,3 & 14,7 \\
\hline $1-9 A$ & 92,49 & 98,19 & 1,04 & 5,53 & 99,11 & 13,9 & 13,9 \\
\hline $1-14 A$ & 53,71 & 58,58 & 1,04 & 5,10 & 91,85 & 20,2 & 20,3 \\
\hline $1-18 B$ & 59,12 & 63,94 & 1,04 & 4,84 & 95,77 & 18,4 & 18,0 \\
\hline $1-19 B$ & 74,10 & 81,03 & 1,04 & 6,82 & 97,84 & 19,7 & 19,8 \\
\hline $1-28$ & 69,37 & 74,51 & 1,04 & 5,17 & 95,71 & 17,2 & 16,7 \\
\hline $1-31 B$ & 78,67 & 83,71 & 1,04 & 4,96 & 97,78 & 14,5 & 14,5 \\
\hline $1-34 A$ & 79,1 & 83,58 & 1,04 & 5,74 & 75,08 & 13,9 & 16,3 \\
\hline
\end{tabular}

Tabela 2 - Particionamento da porosidade por RMN.

\section{Amostra}

$1-2 \mathrm{~A}$

$1-4$

$1-9 \mathrm{~A}$

$1-14 \mathrm{~A}$

1-18B

1-19B

1-28

1-31B

1-34A

\section{Porosidade (\%)}

Micro

8,8

7,7

9,3

5,9

6,2

7,2

9,0

8,7

6,5
Meso

4,0

2,5

2,3

4,2

3,7

4,1

3,5

2,4

2,6
Macro

6,9

4,1

2,3

10,1

8,5

8,4

4,7

3,4

4,8

\section{Porcentagem da porosidade (\%)}

Micro

Meso

Macro

44,5

20,7

34,8

53,6

17,2

29,2

67,0

16,8

16,2

29,1

21,0

49,9

33,4

20,3

46,3

36,6

20,8

42,6

52,3

20,1

27,6

59,7

16,8

23,5

46,7

18,8

34,5 